\title{
THE SCHWARZIAN DERIVATIVE AND SCHLICHT FUNCTIONS
}

\section{ZEEV NEHARI}

It is customary to formulate the inequalities of the "Verzerrungssatz" type for analytic functions $w=f(z)$, schlicht in the unit circle, with reference to a specific normalization. The two normalizations mainly used are: (a) $f(z)$ is finite in $|z|<1, f(0)=0, f^{\prime}(0)=1$; (b) $f(z)$ has a pole at $z=0$ with the residue 1 . If we want to obtain inequalities which are independent of any particular normalization, we have to use quantities which are invariant with regard to an arbitrary linear transformation of the z-plane. The simplest quantity of this type is the Schwarzian differential parameter

$$
\{w, z\}=\left(\frac{w^{\prime \prime}}{w^{\prime}}\right)^{\prime}-\frac{1}{2}\left(\frac{w^{\prime \prime}}{w^{\prime}}\right)^{2},
$$

also called the Schwarzian derivative of $w$ with regard to $z$.

It is easy to obtain an upper bound for $\{w, z\}$ by a simple transformation of the classical inequality $\left|a_{1}\right| \leqq 1$ valid for functions $w=f(z)=z^{-1}+a_{0}+a_{1} z+\cdots$ schlicht in the unit circle. Indeed, applying this inequality to the coefficient of $z$ in the expansion of the schlicht function

$$
\begin{gathered}
g(z)=\frac{f^{\prime}(x)\left(1-|x|^{2}\right)}{f((x+z) /(1+\bar{x} z))-f(x)}=\frac{1}{z}+\bar{x}-\frac{1}{2} \frac{f^{\prime \prime}(x)}{f^{\prime}(x)}\left(1-|x|^{2}\right) \\
-\frac{1}{6}\left(1-|x|^{2}\right)^{2}\left[\left(\frac{f^{\prime \prime}(x)}{f^{\prime}(x)}\right)^{\prime}-\frac{1}{2}\left(\frac{f^{\prime \prime}(x)}{f^{\prime}(x)}\right)^{2}\right] z+\cdots \\
\quad(|x|<1),
\end{gathered}
$$

we obtain $|\{\mid w, z\}| \leqq 6\left(1-|z|^{2}\right)^{-2}$.

We shall now show that by replacing the number 6 in this inequality by 2 , this necessary condition for the schlichtness of $f(z)$ in $|z|<1$ becomes sufficient.

THEOREM I. In order that the analytic function $w=f(z)$ be schlicht in $|z|<1$, it is necessary that

$$
|\{w, z\}| \leqq \frac{6}{\left(1-|z|^{2}\right)^{2}}
$$

Received by the editors May 24, 1948. 
and sufficient that

$$
|\{w, z\}| \leqq \frac{2}{\left(1-|z|^{2}\right)^{2}} .
$$

Proof. By the classical theory of differential equations, the general solution of the differential equation

$$
\{w, z\} / 2=p(z)
$$

is of the form $w=y_{1} / y_{2}$, where $y_{1}$ and $y_{2}$ are two linearly independent solutions of the linear differential equation

$$
y^{\prime \prime}+p(z) y=0 \text {. }
$$

To prove that the condition (2) entails the schlichtness of $w=f(z)$ in $|z|<1$ is therefore equivalent to showing that the ratio $y_{1} / y_{2}$ of two solutions of (4) is schlicht in $|z|<1$ if $p(z)$ is subject to the inequality

$$
|p(z)| \leqq \frac{1}{\left(1-|z|^{2}\right)^{2}}
$$

Now it is easily seen that the schlichtness of $y_{1} / y_{2}$ can be expressed in a different form which is much easier to handle. In order that the ratio of two linearly independent solutions of (4) be schlicht in a certain domain, it is necessary and sufficient that no solution of (4) vanish there more than once. Indeed, if

$$
\frac{y_{1}\left(z_{1}\right)}{y_{2}\left(z_{1}\right)}=\frac{y_{1}\left(z_{2}\right)}{y_{2}\left(z_{2}\right)}=\alpha,
$$

then

$$
y_{1}\left(z_{1}\right)-\alpha y_{2}\left(z_{1}\right)=0, \quad y_{1}\left(z_{2}\right)-\alpha y_{2}\left(z_{2}\right)=0,
$$

that is, the solution $y_{1}(z)-\alpha y_{2}(z)$ of (4) vanishes at the two points $z_{1}$ and $z_{2}$. Our task is therefore reduced to showing that no solution of (4) can vanish in $|z|<1$ more than once if the condition (5) holds.

For this purpose, we multiply equation (4) by $\bar{y} d z$ and integrate from $z_{1}$ to $z_{2}\left(\left|z_{1}\right|<1,\left|z_{2}\right|<1\right)$. This leads to

$$
\int_{z_{1}}^{z_{2}} \bar{y} d\left(y^{\prime}\right)+\int_{z_{1}}^{z_{2}} p|y|^{2} d z=0,
$$

whence we obtain by partial integration

$$
\left[\bar{y} y^{\prime}\right]_{z_{1}}^{z_{2}}-\int_{z_{1}}^{z_{2}}\left|y^{\prime}\right|^{2} \overline{d z}+\int_{z_{1}}^{z_{2}} p|y|^{2} d z=0
$$


the "Green's transform" of (4).

If $y\left(z_{1}\right)=y\left(z_{2}\right)=0$, we have

$$
\int_{z_{1}}^{z_{2}}\left|y^{\prime}\right|^{2} \overline{d z}=\int_{z_{1}}^{z_{2}} p|y|^{2} d z
$$

an identity fundamental in Hille's investigations on the zero-free regions of the solutions of (4) $[1] .^{1}$

In view of the foregoing, Theorem I will be proved if we can show that (6) cannot be true if $p=p(z)$ is subject to the inequality (5). We may obviously confine ourselves to the case in which $\left|z_{1}\right|=\left|z_{2}\right|$ $=\rho<1$ without restricting the generality of the proof. It is even sufficient to show the incompatibility of (5) and (6) for $z_{2}=-z_{1}$, since the condition

$$
|\{w, z\}| \leqq \frac{2}{\left(1-|z|^{2}\right)^{2}}
$$

remains unaltered if $z$ is made subject to any linear transformation $z=k(\alpha-x)(1-\bar{\alpha} x)^{-1}(|\alpha|<1,|k|=1)$ which leaves the unit circle invariant. Indeed, any such transformation can be built up from two rotations of the type $z=k x(|k|=1)$ and a substitution $z=(\beta-x)$ $\cdot(1-\beta x)^{-1}$ with real $\beta$. Now it is easily confirmed that

$$
\{w, x\}=k^{2}\{w, k x\}=k^{2}\{w, z\}
$$

and

$$
\{w, x\}=\frac{\left(1-\beta^{2}\right)^{2}}{(1-\beta x)^{4}}\left\{w, \frac{\beta-x}{1-\beta x}\right\}=\frac{\left(1-\beta^{2}\right)^{2}}{(1-\beta x)^{4}}\{w, z\} .
$$

(8) shows that the substitution $z=k x$ does not affect the inequality

(7). The same is true of the substitution $z=(\beta-x)(1-\beta x)^{-1}$ since, by

(7) and (9),

$$
\begin{aligned}
|\{w, x\}| & =\frac{\left(1-\beta^{2}\right)^{2}}{|1-\beta x|^{4}}|\{w, z\}|<2\left[\frac{1-\beta^{2}}{|1-\beta x|^{2}\left(1-|z|^{2}\right)}\right]^{2} \\
& =2\left[\frac{1-\beta^{2}}{|1-\beta x|^{2}-|\beta-x|^{2}}\right]^{2}=\frac{2}{\left(1-|x|^{2}\right)^{2}}
\end{aligned}
$$

Since, by a substitution $z=k(\alpha-x)(1-\bar{\alpha} x)^{-1}$, any two points $z_{1}, z_{2}\left(\left|z_{1}\right|,\left|z_{2}\right|<1\right)$ may be transformed into two points symmetrical with regard to the origin and neither the schlicht properties of $f(z)$

\footnotetext{
${ }^{1}$ Numbers in brackets refer to the references cited at the end of the paper.
} 
in $|z|<1$ nor condition (7) are affected by this substitution, it is sufficient for our purposes to consider the case $z_{1}=-z_{2}$, where $z_{1}$ may obviously be taken positive. (6) takes in this case the form

$$
\int_{-\rho}^{\rho}\left|y^{\prime}\right|^{2} d r=\int_{-\rho}^{\rho} p|y|^{2} d r \quad(0<\rho<1) .
$$

We shall now use the integral inequality

$$
\int_{-1}^{1} \frac{u^{2} d x}{\left(1-x^{2}\right)^{2}}<\int_{-1}^{1} u^{\prime 2} d x \quad(u=u(x))
$$

which holds for continuous real functions $u(x)$ which vanish at $x= \pm 1$ of the first order. (11) follows easily from the positivedefiniteness of the integral

$$
\int_{-1}^{1}\left(u^{\prime}+\frac{x u}{1-x^{2}}\right)^{2} d x
$$

Expanding and integrating by parts, we obtain

$$
\begin{aligned}
\int_{-1}^{1} u^{\prime 2} d x+\left[\frac{x u^{2}}{1-x^{2}}\right]_{-1}^{1}- & \int_{-1}^{1} \frac{\left(1+x^{2}\right) u^{2}}{\left(1-x^{2}\right)^{2}} d x \\
& +\int_{-1}^{1} \frac{x^{2} u^{2}}{\left(1-x^{2}\right)^{2}} d x>0
\end{aligned}
$$

$u$ being $O(1-x)$ and $O(1+x)$ at $x=1$ and $x=-1$ respectively, the integrals exist and the integrated part vanishes. Hence (11) follows. (11) is an inequality in the strict sense. Equality in (11) would imply the vanishing of the integral (12), whence $u^{\prime}=-x u\left(1-x^{2}\right)^{-1}$, $u=c\left(1-x^{2}\right)^{1 / 2}$. Since this function does not satisfy our hypotheses, equality in (11) is excluded.

Substituting $\rho x$ for $x$ in (11) we obtain the inequality

$$
\rho^{2} \cdot \int_{-\rho}^{\rho} \frac{u^{2} d x}{\left(\rho^{2}-x^{2}\right)^{2}}<\int_{-\rho}^{\rho} u^{\prime 2} d x
$$

valid for functions $u(x)$ possessing zeros of first order at $x= \pm \rho$.

Writing now $y=u+i v$, we have $|y|^{2}=u^{2}+v^{2},\left|y^{\prime}\right|^{2}=u_{x}^{2}+v_{x}^{2}$, and (10) takes the form

$$
\int_{-\rho}^{\rho}\left(u_{x}^{2}+v_{x}^{2}\right) d x=\int_{-\rho}^{\rho} p\left(u^{2}+v^{2}\right) d x .
$$

If $p$ is subject to the inequality (5), we have 


$$
\left|\int_{-\rho}^{\rho} p\left(u^{2}+v^{2}\right) d x\right| \leqq \int_{-\rho}^{\rho} \frac{u^{2}+v^{2}}{\left(1-x^{2}\right)^{2}} d x<\rho^{2} \int_{-\rho}^{\rho} \frac{u^{2}+v^{2}}{\left(\rho^{2}-x^{2}\right)^{2}} d x .
$$

Since $y=u+i v$ is supposed to have zeros of the first order at $z= \pm \rho$, the same is true of $u$ and $v$ separately. Using (13), we obtain therefore

$$
\left|\int_{-\rho}^{\rho} p\left(u^{2}+v^{2}\right) d x\right|<\rho^{2} \int_{-\rho}^{\rho} \frac{u^{2}+v^{2}}{\left(\rho^{2}-x^{2}\right)^{2}} d x<\int_{-\rho}^{\rho}\left(u_{x}^{2}+v_{x}^{2}\right) d x,
$$

which contradicts (14). We have thus proved that no solution of (4) can vanish in $|z|<1$ more than once if $p(z)$ satisfies (5). As shown above, this is equivalent to the schlichtness in $|z|<1$ of the ratio $w=y_{1} / y_{2}$ of two independent solutions of (4). In view of the identity (3), this completes the proof of Theorem I.

Replacing (11) by similar integral inequalities, it is possible to obtain sufficient conditions for the schlichtness of $w=f(z)$ in $|z|<1$ which are different from (2). As an example, we prove the following theorem:

THEOREM II. If

$$
|\{w, z\}| \leqq \pi^{2} / 2
$$

in $|z|<1$, then $w=f(z)$ is schlicht in the unit circle. The constant $\pi^{2} / 2$ in (15) is the best possible.

Proof. As shown above, it is sufficient to prove that the differential equation $y^{\prime \prime}+p y=0$, where $p=\{w, z\} / 2$, cannot have a solution $y$ which vanishes in $|z|<1$ more than once.

Suppose now that $y\left(z_{1}\right)=y\left(z_{2}\right)=0$. Using the identity (6) and taking as the integration path the linear segment connecting $z_{1}$ and $z_{2}\left(z=\left(z_{1}+z_{2}\right) / 2+e^{i \theta} r\right)$, we obtain

$$
\int_{-\rho}^{\rho}\left|y^{\prime}\right|^{2} d r=e^{-2 i \theta} \int_{-\rho}^{\rho} p|y|^{2} d r, \quad 2 \rho=\left|z_{2}-z_{1}\right| .
$$

Writing $y=u+i v$, we have $y^{\prime}=e^{-i \theta}\left(u_{r}+i v_{r}\right)$, whence $|y|^{2}=u^{2}+v^{2}$, $\left|y^{\prime}\right|^{2}=u_{r}^{2}+v_{r}^{2}$. Using this, (16) takes the form

$$
\int_{-\rho}^{\rho}\left(u_{r}^{2}+v_{r}^{2}\right) d r=e^{-2 i \theta} \int_{-\rho}^{\rho} p\left(u^{2}+v^{2}\right) d r .
$$

We now use the inequality

$$
\int_{-\pi / 2}^{\pi / 2} y^{2} d x<\int_{-\pi / 2}^{\pi / 2} y^{\prime 2} d x
$$


[2], which holds for a function $y(x)$ which is continuous for $-\pi / 2$ $\leqq x \leqq \pi / 2$ and has zeros of first order at $x= \pm \pi / 2$. (18) follows immediately from the identity

$$
\begin{aligned}
\int_{-\pi / 2}^{\pi / 2}\left(y^{\prime}+y \operatorname{tg} x\right)^{2} d x & =\int_{-\pi / 2}^{\pi / 2} y^{\prime 2} d x+\left[y^{2} \operatorname{tg} x\right]_{-\pi / 2}^{\pi / 2}-\int_{-\pi / 2}^{\pi / 2} y^{2} d x \\
& =\int_{-\pi / 2}^{\pi / 2} y^{\prime 2} d x-\int_{-\pi / 2}^{\pi / 2} y^{2} d x,
\end{aligned}
$$

which also shows that equality in (18) can only occur for $y=c \cdot \cos x$. Replacing the variable $x$ in (18) by $(\pi / 2 \rho) r$, we obtain

$$
\int_{-\rho}^{\rho} y^{2} d r<\frac{4 \rho^{2}}{\pi^{2}} \int_{-\rho}^{\rho} y^{\prime 2} d r, \quad\left(y^{\prime}=\frac{d y}{d r}\right),
$$

valid for functions $y$ vanishing of first order at $r= \pm \rho$.

Both $u$ and $v$ satisfy these conditions. In view of (3), (15) and (19), we therefore obtain

$$
\begin{aligned}
\left|e^{-2 i \theta} \int_{-\rho}^{\rho} p\left(u^{2}+v^{2}\right) d r\right| & \leqq \frac{\pi^{2}}{4} \int_{-\rho}^{\rho}\left(u^{2}+v^{2}\right) d r \\
& \leqq \rho^{2} \int_{-\rho}^{\rho}\left(u_{r}^{2}+v_{r}^{2}\right) d r<\int_{-\rho}^{\rho}\left(u_{r}^{2}+v_{r}^{2}\right) d_{r} .
\end{aligned}
$$

This contradicts (17) and thus proves Theorem II.

That the constant $\pi^{2} / 2$ in (15) is the best possible is shown by the function $w=f_{0}(z)=e^{\pi i(1+\epsilon) z}, \epsilon>0$. It is easily confirmed that

$$
\{w, z\}=\left(\frac{w^{\prime \prime}}{w^{\prime}}\right)^{\prime}-\frac{1}{2}\left(\frac{w^{\prime \prime}}{w^{\prime}}\right)^{2}=\frac{\pi^{2}}{2}(1+\epsilon)^{2}>\frac{\pi^{2}}{2} .
$$

The period of $f_{0}(z)$ being $2(1+\epsilon)^{-1}<2$, it follows that $f_{0}(z)$ cannot be schlicht in the unit circle.

For the applications it is more convenient to formulate Theorem II in a slightly different way.

Corollary. The radius of univalence of the function $w=f(z)$ is at least equal to the smallest positive root $\rho_{0}$ of the equation

$$
\rho^{2} M(\rho)=\pi^{2} / 2,
$$

where

$$
M(\rho)=\max _{|z|=\rho}|\{w, z\}| .
$$


Indeed, by (3), (15), (19), (20) and (21) we have, for $\rho<\rho_{0}$,

$$
\begin{aligned}
\left|e^{-2 i \theta} \int_{-\rho}^{\rho} p\left(u^{2}+v^{2}\right) d r\right| & \leqq M(\rho) \int_{-\rho}^{\rho}\left(u^{2}+v^{2}\right) d r<\frac{\pi^{2}}{2 \rho^{2}} \int_{-\rho}^{\rho}\left(u^{2}+v^{2}\right) d r \\
& \leqq \int_{-\rho}^{\rho}\left(u_{r}^{2}+v_{r}^{2}\right) d r
\end{aligned}
$$

which again contradicts (17).

As an application of Theorem II, or rather the corollary, we show that the error-function

$$
w=\phi(z)=\int_{0}^{z} e^{-z^{2}} d z
$$

is schlicht in a circle with radius

$$
\rho_{0}=\left(\left(\left(\pi^{2}+1\right)^{1 / 2}-1\right) / 2\right)^{1 / 2}
$$

around the origin. Indeed,

$$
\{w, z\}=-2\left(1+z^{2}\right)
$$

and, by the corollary, the radius of univalence of $\phi(z)$ is therefore at least equal to the positive root of

$$
2 \rho^{2}\left(1+\rho^{2}\right)=\pi^{2} / 2,
$$

that is, (22).

\section{REFERENCES}

1. Einar Hille, Bull. Amer. Math. Soc. vol. 28 (1922) p. 261.

2. G. H. Hardy, J. E. Littlewood, G. Pólya, Inequalities, Cambridge, 1934, p. 184.

HARVARD UNIVERSITY AND

WASHINGTON UNIVERSITY 